\title{
Stand der Technik in der Tagbauvermessung
}

\author{
Alexander Tscharf \\ Lehrstuhl für Bergbaukunde, Bergtechnik und Bergwirtschaft, Montanuniversität Leoben, Leoben, Österreich
}

Eingegangen 17. Oktober 2017; angenommen 23. Oktober 2017; online publiziert 29. Januar 2018

Zusammenfassung: Die bergmännische Vermessung ist ein wichtiger Teil der Mineralrohstoffgewinnung - unter wie auch über Tage. Sie hat die Aufgabe und das Ziel, in allen Phasen des Mineralrohstoffprojekts, beginnend von der Erkundung über die Erschließung und Gewinnung bis hin zur Nachsorge die notwendigen raumbezogenen Daten zu generieren. Die regelmäßige Vermessung des Betriebes ist zur Nachtragung des Bergbaukartenwerks ( $\$ 42$ Markscheideverordnung 2013), zur Massenermittlung sowie auch zur Qualitätssteuerung und als Grundlage aller planerischen Überlegungen unbedingt erforderlich. Im Beitrag werden gängige Methoden der Tagbauvermessung präsentiert, ihre jeweiligen Vor- und Nachteile sowie Einsatzgebiete aufgezeigt und auch der Versuch unternommen, alle Verfahren vor dem Hintergrund der zu erfüllenden Aufgaben gegenüberzustellen. Neben den mittlerweile „klassischen“ Methoden wie GNSS oder Tachymetrie stehen vor allem auch die neuesten Entwicklungen im Bereich der flächigen Aufnahmeverfahren im Zentrum der Ausführungen. Insbesondere wird auch auf technologische Neuerungen und Fortentwicklungen eingegangen und untersucht, inwieweit alternative Verfahren, wie Laserscanning, UAV-Photogrammetrie oder Radar, eine Ergänzung oder in gewissen Bereichen auch Ersatz bestehender und etablierter Lösungen sein können.

Schlüsselwörter: Markscheidewesen, Tagbauvermessung, GNSS, Videotachymeter, Laser Scanning, Radar,

Photogrammetrie

State of the Art in Open Pit Mine Surveying

Abstract: Mine surveying with the aim to provide the necessary geospatial data in all phases of the mineral resource

Nach einem Vortrag, gehalten am Österreichischen Bergbautag in Bad Ischl, Oberösterreich, 9. bis 11. Mai 2017

Dipl.-Ing. A. Tscharf ( $\bowtie)$

Lehrstuhl für Bergbaukunde, Bergtechnik und Bergwirtschaft,

Montanuniversität Leoben,

Franz-Josef-Str. 18,

8700 Leoben, Österreich

alexander.tscharf@unileoben.ac.at project is an essential part of mineral resource extraction. The regular surveying of the mining operation is absolutely necessary for updating the mining maps ( $\$ 42$ Markscheideverordnung 2013), for quantity surveys as well as for quality control and as a basis for all planning considerations. This paper presents the current methods of open pit mine surveying, their respective advantages and disadvantages as well as areas of application, and especially in the light of tasks to be fulfilled all methods are compared with each other.

In addition to the "classical" methods such as GNSS or tachymetry, the focus lies on the latest developments in the field of areal surveying techniques. In particular, technological innovations and new developments are investigated and it is examined to what extent alternative methods, such as laser scanning, UAV photogrammetry or radar, can be a supplement or, in certain areas, a replacement for existing and established solutions.

Keywords: Mine Surveying, GNSS, Image assisted total station, Laser scanning, Radar, Photogrammetry

\section{Markscheiderische Aufgaben im Tagbau}

Zur Gewinnung mineralischer Rohstoffe im Tagbau kommen im Allgemeinen Verfahren der Trockengewinnung, der Nassgewinnung und der hydraulischen Gewinnung zum Einsatz, wobei jeweils spezielle markscheiderische Arbeiten erforderlich sind. Die Hauptaufgaben, die der verantwortliche Markscheider unter Berücksichtigung der im Mineralrohstoffgesetz (MinroG [1]) beschriebenen Aufgaben zu bearbeiten hat, sind bei allen genannten Verfahren vergleichbar und umfassen Tätigkeiten von der Gewinnungsvorbereitung über die tatsächliche Gewinnung und Produktionskontrolle bis hin zur Nachsorge und Beweissicherung. Konkret führt § 135 des Mineralrohstoffgesetzes [1] die folgenden Punkte an:

1. Das Anfertigen und die Führung des Bergbaukartenwerks,

2. Die Beaufsichtigung der Vermessung im Bergbau, 
3. Aufgaben der bergbaulichen Raumordnung und Sicherungspflicht und

4. Bergschadenkundliche Aufgaben, vor allem zum Schutz der Oberfläche und zur Sicherung der Oberflächennutzung nach Beendigung der Bergbautätigkeit.

Das Anfertigen und Führen des Bergbaukartenwerks stellt wahrscheinlich den wesentlichsten Teil der markscheiderischen Aufgaben dar. Der verantwortliche Markscheider ist verantwortlich für die Richtigkeit, die Genauigkeit und die Vollständigkeit (§ 110 MinroG [1]) des Bergbaukartenwerks, welches alle Risse, Karten und Pläne des Bergbaues umfasst. Im Tagbau sind dies im Wesentlichen die Lagepläne, Tagbaugrundrisse, Bodenbewegungsrisse, Rekultivierungspläne, schnittrissliche Darstellungen und Detailpläne. Zusätzlich sind alle Bescheide sowie Vermessungs- und Berechnungsprotokolle Teile des Bergbaukartenwerks.

Die Vermessung bzw. die Beaufsichtigung der Vermessung im Bergbau obliegt ebenso dem verantwortlichen Markscheider. Vermessungsergebnisse müssen rekonstruierbar dokumentiert werden und die Richtigkeit der Ergebnisse und der darauf basierenden graphischen Darstellungen müssen gewährleistet sein.

Aufgrund der Ortsgebundenheit des Bergbaus kommt der bergbaulichen Raumordnung in enger Wechselwirkung mit der örtlichen Raumplanung große Bedeutung zu. Mindestabstände zu anderen Flächenwidmungen müssen eingehalten werden und ganz grundsätzlich müssen konkurrierende Raumnutzungen in die Planungsarbeiten miteinbezogen werden. Die bergbauliche Sicherungspflicht umfasst den Schutz von Leben und Gesundheit von Personen, den Schutz von fremden Sachen, der Umwelt und der Oberfläche sowie den Lagerstättenschutz und die Sicherung der Oberflächennutzung nach Beendigung der Bergbautätigkeit.

Im Tagbau sind die bergschadenkundlichen Aufgaben nicht so umfangreich wie im untertägigen Bergbau. Sie konzentrieren sich im Wesentlichen auf die Standsicherheit von Böschungen, wobei die Böschungsstabilität messtechnisch erfasst und gegebenenfalls Standsicherheitsnachweise durchgeführt werden müssen.

Die regelmäßige Vermessung des Tagbaus und der Abbaustände ist zur Erfüllung der genannten Aufgaben unbedingtes Erfordernis. Sie dient zur Nachtragung des Bergbaukartenwerks und darüber hinaus bildet sie die Basis jeglicher bergmännischen Planungs- und Betriebstätigkeit, indem sie die Grundlage für die Berechnung der bewegten Abraum- und Rohstoffmengen, aber insbesondere auch zur Qualitätssteuerung liefert. Die Tagbauvermessung vermittelt somit nicht nur den aktuellen Stand der Abbausituation, sondern gibt auch Auskunft über vorhandene Böschungssysteme und Böschungswinkel sowie Arbeitsebenen inklusive vorhandener Band- und Gleisanlagen, sowohl in Lage und Höhe, als auch in Beziehung zueinander [2].

In den nachfolgenden Ausführungen werden gängige und neue Methoden bei der Durchführung der operativen Tagbauvermessung bzw. Beweissicherungsmessung präsentiert. Die jeweiligen Vor- und Nachteile sowie Einsatzgebiete werden aufgezeigt und es wird auch der Versuch unternommen, alle Verfahren vor dem Hintergrund der zu erfüllenden Aufgaben gegenüberzustellen.

\section{Erforderliche Genauigkeiten}

Dem jeweiligen Zweck entsprechend gibt die österreichische Markscheideverordnung [3] unterschiedliche Genauigkeiten für Lage und Höhe an, wobei sich die höchsten Genauigkeiten auf Vermessungen im Zusammenhang mit Bodenbewegungen ( $\$ 43$ ) beziehen [3].

Die Verordnung sieht die Erfassung von Bodenbewegungen durch Lage- und Höhenmessungen unter anderem dann vor, wenn im Tagbau Böschungsbewegungen auftreten bzw. zu erwarten sind. Ebenso in Bereichen von Bergbauhalden oder -dämmen bzw. in geologisch labilen Zonen. Bei Lagemessungen ist dann eine Genauigkeit von $\pm 0,02 \mathrm{~m}$, bei Höhenmessungen von $\pm 10 \mathrm{~mm} / \mathrm{km}$ einzuhalten. Die Messungen sind nach dem Stand der Wissenschaft und Technik geeigneter Methoden durchzuführen und überdies durch eine gleichwertige andere Methode oder, sofern dies nicht möglich ist, nach der gleichen Methode zu sichern [3].

Für allgemeine Tagbauvermessungen kommen die Paragrafen $15 \mathrm{ff}$ zur Anwendung (insbesondere § 15 und § 18 Markscheideverordnung, 2013). Demnach müssen Vermessungen über Tage unter Anwendung nach dem Stand der Technik geeigneter Methoden sowie unter Einhaltung der in Abs. 2 genannten Genauigkeitsanforderungen durchgeführt werden [3].

$\S 15$ [2] regelt die Bestimmung bergbaueigener Festpunkte. Vermessungen über Tage sind demnach so vorzunehmen, dass unter Bedachtnahme auf die mittlere Punktlagegenauigkeit der Festpunkte der Landesvermessung (Triangulierungspunkte $\pm 0,05 \mathrm{~m}$, Einschaltpunkte $\pm 0,07 \mathrm{~m}$ ) die nachstehend angegebene mittlere Punktlagegenauigkeit für die bergbaueigenen Festpunkte eingehalten wird:

1. bei der Bestimmung von Standpunkten: $\pm 0,10 \mathrm{~m}$ und

2. bei der Bestimmung anderer Festpunkte: $\pm 0,15 \mathrm{~m}$.

Gem. Abs. 3 ist der höhenmäßige Anschluss an das System der Landesvermessung (Triangulierungspunkte, Höhenpunkte des Präzisions- und nachgeordneten Nivellements) so durchzuführen, dass eine Höhengenauigkeit von $\pm 0,10 \mathrm{~m}$ eingehalten wird [3].

Allgemeine Tagbauvermessungen sind gem. § 18 nach den technischen und geologisch-lagerstättenkundlichen Gegebenheiten entsprechenden Methoden durchzuführen, wobei sowohl bei Lage- als auch Höhenmessungen eine Genauigkeit von mindestens $\pm 0,20 \mathrm{~m}$ einzuhalten ist [3].

Die genannten Genauigkeiten stellen Mindestanforderungen dar, die auch bei der Gewinnung von mineralischen Rohstoffen etwa in der Nasskiesgewinnung gültig sind, sofern nicht andere behördlich, im Bewilligungsbescheid angeordnete Vorgaben größere Genauigkeiten erforderlich machen [3]. 


\section{Methoden}

Gemäß den gesetzlichen Vorgaben (MinroG i. V.m. Markscheideverordnung 2013) sind Vermessungsarbeiten nach dem Stand der Technik durchzuführen. Dieser unterliegt jedoch insbesondere durch die zunehmende Digitalisierung, die Miniaturisierung von verfügbarer Sensorik und das stetige Aufkommen neuer Technologien einer permanenten Veränderung, weshalb im Folgenden der heutige Stand der Technik bei der Tagbauvermessung abgebildet werden soll. Dabei muss jedoch berücksichtigt werden, dass lediglich die gängigsten bzw. wichtigsten Methoden dargestellt, allzu tiefgreifende technische Details aufgrund der starken Anwendungsfokussierung ausgespart werden und kein Anspruch auf absolute Vollständigkeit erhoben wird.

\subsection{Global Navigation Satellite System (GNSS)}

In etwa seit den 1960er-Jahren werden Satellitensysteme zur Positionsbestimmung und weltweiten Navigation aufgebaut. Dabei werden grundsätzlich Distanzen bzw. Distanzänderungen zwischen Satelliten (Raumsegment) und Stationen auf der Erde (Benutzersegment) gemessen, woraus in weiterer Folge dreidimensionale Koordinaten der Stationen abgeleitet werden können. Die Positionsbestimmung erfolgt somit - vereinfacht dargestellt - als dreidimensionaler Bogenschnitt aus zumindest vier gemessenen Distanzen (drei Satelliten zur Lagebestimmung und ein Satellit zur Korrektur von Laufzeitfehlern bzw. zur Synchronisation der Uhren). Die Beobachtung kann statisch (mit ruhendem Empfänger) oder kinematisch (mit bewegtem Empfänger) erfolgen, wobei die verschiedenen Verfahren zusätzlich auch noch nach der Anzahl der zum Einsatz kommenden Satellitenempfänger unterschieden werden können $[4,5]$.

Aus dem Umstand, dass die Bodenstation Signale der Satelliten empfangen muss, ergibt sich eine der signifikantesten Einschränkungen bei der praktischen Anwendung satellitengestützter Verfahren (vgl. Tab. 1): Der zu messende bzw. zu beobachtende Punkt muss zugänglich sein, um den Satellitenempfänger auch tatsächlich dort zu positionieren, was jedoch insbesondere im Bergbau aus topographischen oder sicherheitlichen Gründen nicht immer möglich ist.

Steht nur ein Empfänger zur Verfügung, ist nur eine Einzelpunktbestimmung möglich. Durch zahlreiche Fehlereinflüsse (z. B. Refraktion, Satellitenbahnfehler, Multi-Path-Effekte) wird jedoch in der Regel nur eine geringe Genauigkeit erreicht. Eine verbesserte kinematische Einzelpunktbestimmung in Echtzeit kann durch das sogenannte Differentielle GNSS erreicht werden, wo zumindest zwei Empfänger benötigt werden, wovon einer als koordinativ bekannte Referenzstation und einer als bewegter "Rover" verwendet wird [5].

Werden mit mehreren Empfängern gleichzeitig dieselben Satelliten beobachtet, so spricht man von relativer Punktbestimmung. Die Genauigkeit der relativen Punktbestimmung ist im Vergleich zur Einzelpunktbestimmung wesentlich höher, da durch die Kombination der Beobachtungsdaten von mehreren Punkten Fehlereinflüsse ausge-

\begin{tabular}{|ll|}
\hline $\begin{array}{l}\text { TABELLE } 1 \\
\text { Vor- und Nachteile GNSS Vermessung }\end{array}$ \\
\hline Vorteile & Nachteile \\
\hline $\begin{array}{l}\text { Einfach und schnell einsetz- } \\
\text { bar }\end{array}$ & $\begin{array}{l}\text { Geringere Genauigkeit } \\
(\sim 5 \mathrm{~cm})\end{array}$ \\
\hline $\begin{array}{l}\text { Keine Anschlussvermes- } \\
\text { sung notwendig }\end{array}$ & $\begin{array}{l}\text { Abschattungseffekte (spezi- } \\
\text { ell bei tiefen Aushebungen) }\end{array}$ \\
\hline $\begin{array}{l}\text { Automatisiertes Monitoring } \\
\text { möglich }\end{array}$ & $\begin{array}{l}\text { Auswertung gleicht gewis- } \\
\text { sermaßen einer "Black Box" }\end{array}$ \\
\cline { 2 - 2 } & \begin{tabular}{l} 
Verbindungsprobleme \\
\cline { 2 - 2 }
\end{tabular} \\
$\begin{array}{l}\text { Punkt muss begangen wer- } \\
\text { den }\end{array}$ \\
\hline
\end{tabular}

schaltet werden können. Die Auswertung der Basislinien der relativen Punktbestimmung erfolgt in der Regel nach der Messung im Innendienst. Soll die Punktbestimmung jedoch in Echtzeit durchgeführt werden, so müssen die Beobachtungsdaten der einen Station zur anderen übertragen werden, wie es bei der häufigsten GNSS-Anwendung, dem sogenannten Real-Time-Kinematic (RTK) GNSS, der Fall ist $[4,5]$.

\subsubsection{Praktische Gesichtspunkte}

Nicht zuletzt aufgrund der vielseitigen Navigationsanwendungen (Fahrzeuge, Schiffe, Smartphones, ...) nimmt GNSS-Vermessung auch in der markscheiderischen Tagbauvermessung einen zunehmend hohen Stellenwert ein. In der täglichen Anwendung ist die Komplexität des Systems aus Raum-, Kontroll- und Nutzersegment samt notwendiger Referenzstationen und Korrekturdaten kaum bemerkbar und GNSS-Systeme sind auch für nicht speziell ausgebildete Anwender schnell und einfach einsetzbar. Durch die Nutzung von extraterrestrischen Satelliten ist überdies eine absolute Positionsbestimmung möglich und eine Anschlussvermessung an das System der Landesvermessung im herkömmlichen Sinne kann (zumindest im Feld) entfallen.

Nichtsdestotrotz müssen die aufgenommenen Rohdaten nach der Aufnahme prozessiert und verarbeitet werden, wobei eine hundertprozentige Nachvollziehbarkeit durch den Anwender nicht immer in vollem Ausmaß gegeben ist (Black-Box-Charakter). Die Tatsache, dass zur Bestimmung der Koordinaten der zu vermessende Punkt mit dem Satellitenempfänger begangen werden muss, kann im Bergbau aus zweierlei Gründen problematisch sein. Einerseits ist der Zugang zu Punkten aus sicherheitlichen und topographischen Gründen nicht immer möglich, und andererseits kann die spezielle Geometrie eines typischen Tagbaus sehr oft Signalabschattungen zu den Satelliten bewirken, wodurch die Messung in weiterer Folge gar nicht durchführbar wird. Ein Umstand, der insbesondere bei tiefen Aushebungen nicht außer Acht gelassen werden darf.

Hinsichtlich erreichbarer Genauigkeit ist anzumerken, dass diese bei der - abgesehen von Deformationsbeobachtungen - zumeist angestrebten Echtzeitlösung in der Größenordnung weniger Zentimeter liegen kann, wodurch das Verfahren zur Durchführung von gängigen Tagbauvermessungen auf jeden Fall den Anforderungen genügt 
und bei gut geplanten, statischen Messkampagnen mit entsprechender Beobachtungszeit durchaus auch zur Erfassung von Bodenbewegungen geeignet ist.

\subsection{Videotachymeter (Image Assisted Total Station, IATS)}

Die Aufnahmemethode zur Erschließung von Bildinformationen durch die Verbindung von reflektorlos messenden und ferngesteuerten Tachymetern mit Digitalkameras wird als Phototachymetrie bezeichnet. Bei diesem Messverfahren werden Elemente der Tachymetrie und der Photogrammetrie verknüpft mit dem Ziel, die Geometrie des Tagbaus oder Teile davon zu bestimmen und gleichzeitig das aufgenommene Objekt zu visualisieren. Die Aufnahme erfolgt in Blickrichtung des Tachymeterfernrohrs, was durch den Einbau von Kameras in das Tachymeter realisiert wird [2].

Nahezu alle modernen Totalstationen (aller Hersteller) sind mittlerweile mit integrierten Fernrohr- bzw. Weitwinkelkameras ausgestattet. Abzusteckende oder bereits aufgenommene Punkte können in das Live-Bild eingetragen werden, und mittels integrierter Bildverarbeitung bzw. Scannerfunktionalität lassen sich schnell 3D-Modelle ableiten, welche in weiterer Folge zum Beispiel zur Volumsund Massenermittlung herangezogen werden können. Die Steuerung der "Robotik-Instrumente" ist in der Regel direkt aus dem Bild heraus möglich, automatische Zielerfassung kann mit reflektorloser Entfernungsmessung kombiniert werden und der Ein-Mann-Betrieb ist somit verhältnismäBig robust realisiert.

\subsubsection{Praktische Gesichtspunkte}

Videotachymeter verknüpfen „klassische“ Tachymetrie mit photogrammetrischer Aufnahme und Scanning-Funktion. Aufgrund der Tatsache, dass die Basis dieser Instrumente grundsätzlich nach wie vor durch einen Theodolit gebildet wird, bieten sie auch die damit einhergehende Vielfalt an Anwendungsmöglichkeiten. Unter Bezugnahme auf die zu Beginn beschriebenen Aufgaben in der Tagbauvermessung ist das Tachymeter somit wohl nach wie vor jenes Instrument, mit dem die meisten Aufgaben erfüllt werden können.

Neben der reinen Erfassung der Örtlichkeit, was durch reflektorlose Messung auch bei nicht zugänglichen Punkten möglich ist, können auch Absteckungen vorgenommen und Planungen in die Örtlichkeit übertragen werden. Überdies zählen vergleichbare Instrumente zu den genauesten Möglichkeiten, geodätische Beobachtungen durchzuführen, und durch den Einbau von Servomotoren sind sie außerdem ideal für automatisierte Monitoringlösungen geeignet.

Nachteilig sticht die Tatsache einer notwendigen Sichtverbindung zwischen Stand- und Zielpunkt hervor. Sind aus topographischen Gründen nur kurze Visurlängen möglich, so müssen linienhafte Verfahren der Neupunktbestimmung (Polygonzug) angewandt werden, und es sind entsprechend viele Aufstellungen vonnöten. In Tab. 2 werden die wichtigsten Vor- und Nachteile der Videotachymetrie

\begin{tabular}{|c|c|}
\hline Vorteile & Nachteile \\
\hline $\begin{array}{l}\text { "Einfach" und schnell in der } \\
\text { Anwendung }\end{array}$ & Sichtprobleme \\
\hline Hochgenau $(<5 \mathrm{~mm})$ & Verhältnismäßig teuer \\
\hline $\begin{array}{l}\text { Reflektorlose Messung mög- } \\
\text { lich }\end{array}$ & Umgang sollte gelernt sein \\
\hline $\begin{array}{l}\text { Automatisiertes Monitoring } \\
\text { möglich }\end{array}$ & \\
\hline $\begin{array}{l}\text { Simultane Verwendung der } \\
\text { Bildinformation }\end{array}$ & \\
\hline $\begin{array}{l}\text { Nahezu alle Aufgaben in der } \\
\text { Tagbauvermessung können } \\
\text { erfüllt werden }\end{array}$ & \\
\hline
\end{tabular}

noch einmal übersichtlich zusammengefasst, wodurch der Vergleich mit anderen Verfahren vereinfacht wird.

\section{3 "Terrestrisches" Laser Scanning}

In der Entwicklung geodätischer Vermessungsinstrumente stellen Laserscanner den Übergang von diskreten zu kontinuierlichen Messungen dar und haben nicht nur bei Anwendungen im Bergbau richtungsweisende Veränderungen mit sich gebracht. Statt einiger weniger ausgewählter Punkte wird ein Objekt ganzheitlich und flächig sowie reflektorlos und ohne Signalisierung von Zielpunkten erfasst. Während bei der tachymetrischen Aufnahme immer diskrete Punkte eingemessen werden, für die als Primärdaten Horizontal- und Vertikalwinkel sowie Schrägdistanzen bestimmt werden, erfolgt bei den flächigen Verfahren, wie Laserscanning und Photogrammetrie, eine flächenhafte Erfassung des gesamten sichtbaren Geländes. Grundsätzlich kann die Aufnahme mittels Laserscanner auch als sehr dichte Polaraufnahme betrachtet werden. Ein Laserstrahl wird gezielt abgelenkt, die Ablenkwinkel werden gemessen, und zusätzlich zur Erfassung der Objektgeometrie aus den gemessenen Winkeln und Distanzen wird zumeist auch noch die Intensität der reflektierten Signale registriert [2].

Während traditionell zwischen „Airborne Laserscanning" (von Flugzeugen oder Hubschraubern aus) und "terrestrischem Laserscanning" unterschieden wird, gewinnen zunehmend auch mobile Plattformen, wie Fahrzeuge, Schiffe, unbemannte Flugsysteme oder auch handgetragene Systeme zur Datenerfassung an Bedeutung. Diese Entwicklung ist hauptsächlich auf die Miniaturisierung und die entsprechenden Fortentwicklungen im Bereich der Informationstechnologie zurückzuführen und wird unter dem Gesichtspunkt des autonomen Fahrens bzw. der autonomen Maschinensteuerung auch künftig einer stetigen Weiterentwicklung unterliegen. In diesen Fällen wird zumeist von kinematischem Laserscanning bzw. von „mobile mapping" gesprochen. Aus heutiger Sicht stellen diese Verfahren eine wertvolle Ergänzung zu bestehenden Lösungen - insbesondere zur Inspektion und Dokumentation - dar, auch wenn die erreichbare Genauigkeit der mobi- 


\begin{tabular}{|ll|}
\hline \multicolumn{2}{|l|}{$\begin{array}{l}\text { TABELLE } 3 \\
\text { Vor- und Nachteile Laser Scanning }\end{array}$} \\
\hline Vorteile & Nachteile \\
\hline $\begin{array}{l}\text { Vollständige 3D Daten- } \\
\text { erfassung }\end{array}$ & Große Datenmengen \\
\hline Hochgenau & Zeitintensives Post-processing \\
\hline $\begin{array}{l}\text { Kombinierte Nutzung } \\
\text { mit Kameras möglich }\end{array}$ & Schweres und teures Equipment \\
\cline { 2 - 2 } & Schrittweise Datenakquisition \\
\hline
\end{tabular}

len Anwendungen auf jeden Fall um eine Größenordnung geringer zu erwarten ist als jene der stationären.

\subsubsection{Praktische Gesichtspunkte}

Im Gegensatz zu photogrammetrischen Verfahren, wo das Objekt aus zumindest zwei Standpunkten aufgenommen werden und zusätzlich auch noch eine Skalierung bzw. Georegistrierung erfolgen muss, ist bei der Verwendung von Laserscannern und ausreichender Einsehbarkeit des Objekts ein Instrumentenstandpunkt zur vollständigen 3D-Datenerfassung ausreichend. Bei größeren Arealen ist es jedoch oftmals notwendig, mehrere Aufnahmen von mehreren Standpunkten mittels Passpunkten zu verknüpfen, um insbesondere eine regelmäßige Punktdichte zu gewährleisten. Treffen die Laserstrahlen in schleifenden Schnitten auf eine geneigte Fläche, entsteht in Abhängigkeit von der Ebenenneigung und dem Abstand des Scanners eine projektiv verzerrte, weitmaschige Punktanordnung mit unregelmäBiger Punktdichte.

Die erreichbare Genauigkeit der Einzelpunkte ist vor allem im Vergleich zu anderen flächenhaften (Nahbereichs-) Verfahren wie der modernen auf "Structure from Motion" basierenden Photogrammetrie sehr hoch. Durch die Kombination mit Kameras bzw. auch anhand der registrierten Intensitätswerte kann eine vollständige und bildhafte Darstellung erreicht werden (vgl. Tab. 3).

Obwohl das terrestrische Laserscanning in der Tagbauvermessung gegenüber den klassischen Verfahren wie z. B. der Tachymetrie einige Vorteile aufweist, wird von vielen Anwendern das notwendige Post-processing neben den hohen Anschaffungskosten und den nicht immer erforderlichen großen Datenmengen als größter Nachteil empfunden (vgl. Tab. 3).

\subsection{Radar}

Radar - Radio Detection And Ranging - steht als Definition gleichsam für die Technik wie auch für ein Gerät zur Entfernungsmessung mittels hochfrequenter Signale. Ursprünglich zumeist im Bereich der Satellitenfernerkundung eingesetzt, ist Radar auch im terrestrischen Bereich in vielen Gebieten anzutreffen (z. B.: bei Bewegungsmeldern, Einparkhilfen, Geschwindigkeitsmessungen, ...). Doch auch zur kleinräumigen Böschungsbeobachtung und zur Analyse von Strukturveränderungen und Risiken bietet die terrestrische Radarinterferometrie eine wichtige Alternative zu satellitengestützten Anwendungen. Die aktiven Radarinstrumente senden Mikrowellenimpulse aus und empfan- gen gleichzeitig die rückgestreuten Signale. Dabei werden die Amplitude, die Phase sowie die Polarisation der reflektierten Strahlung registriert und als sogenannte Amplitudenbilder gespeichert $[6,7]$.

Ein SAR-Amplitudenbild stellt prinzipiell, vergleichbar mit einer photographischen Aufnahme, nur eine zweidimensionale Abbildung des Objekts dar. Das in den $\mathrm{Pi}$ xeln gespeicherte Rückstreuecho ist mehrdeutig, was eine dreidimensionale Positionsbestimmung zunächst unmöglich macht. Deshalb wird bei der Radarinterferometrie die Erdoberfläche von zwei leicht unterschiedlichen Aufnahmepunkten betrachtet und pixelweise die Phasendifferenz zwischen den beiden Aufnahmen berechnet. Diese Phasendifferenz ist dem Wegunterschied des erkannten Objekts proportional und setzt sich aus mehreren Komponenten zusammen [6].

Für Anwendungen in der Tagbauvermessung im typischen österreichischen Maßstab liegt die größte Bedeutung von Radarsensorik also wohl im Einsatz zur Erfassung von Bodenbewegungen mittels terrestrischer Radarinterferometrie. Unter Verwendung von terrestrischen Radarsystemen können relative Verschiebungen über große Distanzen in kleinen bis mittelgroßen Gebieten erfasst werden, wobei auch ein automatisierter Monitoringbetrieb realisierbar ist.

\subsubsection{Praktische Gesichtspunkte}

Einer der hervorstechendensten anwendungsorientierten Vorteile der Radarbeobachtung ist neben der erreichbaren Genauigkeit von wenigen Millimetern vor allem die universelle Einsetzbarkeit bei allen Witterungs- und Lichtbedingungen. Radar ist nachtsichtaktiv und weitestgehend witterungsunabhängig. Eine hochfrequente und simultane Datenerfassung ist möglich, Geometrieveränderungen können auch in großen Entfernungen (z. B. vom Gegenhang) detektiert werden.

Dem gegenüber steht jedoch ein komplexes Post-processing der Daten, eine im Gegensatz zu Laserscanning und Photogrammetrie geringere räumliche Auflösung sowie die Tatsache, dass Deformationen nur in "Line of Sight", also in Blickrichtung des Sensors erfassbar sind. Zudem können bei starken Veränderungen zwischen zwei Messepochen Mehrdeutigkeiten auftreten, die eine eindeutige Aussage oft signifikant erschweren.

Die Tatsache, dass es sich bei terrestrischen Radarsystemen "noch" um ein sehr teures Verfahren handelt, wird sich wohl wie bei allen beschriebenen Methoden in den nächsten Jahren aufgrund des stärkeren Marktdrucks und der entstehenden Konkurrenzsituationen ändern, weshalb dieser Nachteil mit hoher Wahrscheinlichkeit bald als vernachlässigbar angesehen werden kann.

Hinsichtlich der Erfüllung der markscheiderischen Aufgaben in der Tagbauvermessung stellt Radar jedoch bestenfalls eine Ergänzung zu den zuvor genannten Systemen wie GNSS oder Tachymetrie dar. Aufgrund der vorhandenen Schwächen im „Mapping“ können Nachtragsvermessungen nur bedingt bzw. mit erhöhtem Aufwand, und Absteckungen oder die Herstellung bzw. Verdichtung des berg- 


\begin{tabular}{|ll|}
\hline $\begin{array}{l}\text { TABELLE } 4 \\
\text { Vor- und Nachteile Radar }\end{array}$ \\
\hline Vorteile & Nachteile \\
\hline $\begin{array}{l}\text { Simultane und hochfre- } \\
\text { quente Datenerfassung }\end{array}$ & $\begin{array}{l}\text { Mehrdeutig bei starken Verän- } \\
\text { derungen }\end{array}$ \\
\hline $\begin{array}{l}\text { Hochgenaue Erfassung } \\
\text { von Geometrieverände- } \\
\text { rungen in großen Entfer- } \\
\text { nungen }\end{array}$ & Komplexes Post-processing \\
\hline Nachtsichtaktiv & Standortgebunden \\
\hline & $\begin{array}{l}\text { Geringe räumliche Auflösung } \\
\text { Deformationen nur in Blickrich- } \\
\text { tung erfassbar }\end{array}$ \\
\hline
\end{tabular}

baueigenen Festpunktfeldes gar nicht durchgeführt werden (Tab. 4).

\subsection{Photogrammetrie/UAV-Photogrammetrie}

Photogrammetrische Verfahren werden bereits seit Jahrzehnten in den verschiedensten Bereichen der markscheiderischen Vermessung eingesetzt. Auch in der Tagbauvermessung finden sowohl Luftbilder, als auch terrestrische Detailaufnahmen, z.B. bei der Bruchwandvermessung im Rahmen der Sprengplanung, Anwendung. Aus zumindest zwei Bildern wird basierend auf gefundenen Korrespondenzen ein 3D-Modell des Objekts berechnet, welches neben der reinen Geometrieinformation auch eine bildhafte Interpretation durch den Anwender ermöglicht.

Hervorgerufen durch die rasanten Entwicklungen im Bereich der Robotik und vor allem auch der Computer Vision und digitalen Bildverarbeitung erlebt die Photogrammetrie jedoch derzeit eine technologische Renaissance, und bildbasierte Verfahren finden wieder vermehrt Eingang in die vermessungstechnische Forschung, Entwicklung und auch Anwendung. Moderne Verfahren (Structure from Motion) sind in der Lage, aus einer Vielzahl an (zunächst ungeordneten) Bildern die äußere Orientierung der Kamera und eine punktbasierte Szenenrekonstruktion zu errechnen. Die einzige Voraussetzung ist ein ausreichender Bildüberlapp, um die Korrespondenzanalyse zwischen den unterschiedlichen Aufnahmen zu ermöglichen [2, 8].

Einen besonderen Stellenwert im Zusammenhang mit photogrammetrischen Verfahren nimmt die Verwendung von unbemannten Flugsystemen (UAS bzw. UAV) als Trägerplattform ein. Kleine und leicht zu steuernde unbemannte Flugsysteme sind in der Lage, die Lücke zwischen luftgebundenen und terrestrischen Verfahren zu schließen, wodurch sich viele für die Mineralrohstoffgewinnung relevante Anwendungspotentiale auftun, die bei [8] im Detail beschrieben sind.

\subsubsection{Praktische Gesichtspunkte}

Während die klassische Luftbildphotogrammetrie durch den Einsatz von bemannten spezialisierten Flugzeugen hohe Kosten verursacht und dementsprechend nur für große Tagbaugebiete wirtschaftlich durchführbar ist, kann die terrestrische Photogrammetrie auch in kleineren Betrieben für verschiedene Anwendungen eingesetzt werden.

Besonders die Nutzung von UAVs zur photogrammetrischen Bildaufnahme ist durch eine hohe Flexibilität, Sicherheit, Vollständigkeit und (zumindest im Feld) Wirtschaftlichkeit gekennzeichnet. Überdies stellt die vorhandene Bildinformation neben der erfassten Geometrie eine wertvolle zusätzliche Informationsquelle dar. Diese könnte einerseits Basis einer automatisierten Interpretation zur Erkennung markanter Merkmale sein, wie z. B. Eigenschaften von gesprengtem Hauwerk oder auch standsicherheitsrelevante Elemente an Böschungen. Andererseits bieten Bilder auch immer eine gute Grundlage zur Interpretation durch menschliche Anwender und verbinden so Vermessung und Geometrieerfassung unmittelbar mit Inspektion und Interpretation.

Die Kehrseite der sehr schnellen Datenerfassung ist mit Sicherheit die aufwendigere, weil zeit- und vor allem rechenintensive, Auswertung. Während der zeitliche Aufwand zur Vermessung im Feld durch die weitestgehend autonome Datenerfassung reduziert wird, stellt die weitere Verarbeitung der Daten sowie die Integration in gewohnte Arbeitsabläufe (Erzeugung gängiger Produkte aus neuen Datenquellen) eine nicht zu unterschätzende Herausforderung dar. Eine praktische Einschränkung ergibt sich wiederum in Form von Bewuchs. Naturgemäß geben bildgebende Verfahren die Realität ohne jegliche Interpretation und Filterung wieder, und das so abgeleitete Oberflächenmodell enthält somit auch bewachsene Flächen, die in der Regel interaktiv nachbearbeitet werden müssen. Außerdem wirken sich äußere Einflüsse wie Witterung und Sonnenstand wesentlich stärker auf die fotografische Bildaufnahme aus als zum Beispiel auf die Vermessung mittels Laserscanner oder Tachymetrie (Tab. 5).

\section{Gegenüberstellung und Schluss- folgerungen}

Zusammenfassend zeigt sich, dass alle präsentierten bzw. gängigen Verfahren in gewissen Bereichen Stärken und in anderen wiederum Schwächen aufweisen. Vor dem Hintergrund der zu erfüllenden markscheiderischen Aufgaben im Tagbau kann Abb. 1 eine grafische Übersicht entnommen werden. Wird auch die erreichbare Genauigkeit in die Betrachtung miteinbezogen, so zeigt sich, dass das "klassische Tachymeter" trotz aller Neuentwicklungen nach wie

\begin{tabular}{|ll|}
\hline $\begin{array}{l}\text { TABELLE } 5 \\
\text { Vor- und Nachteile Photogrammetrie }\end{array}$ \\
\hline Vorteile & Nachteile \\
\hline Flexibilität & Große Datenmengen \\
\hline Sicherheit & $\begin{array}{l}\text { Problematisch in Bereichen } \\
\text { mit Bewuchs }\end{array}$ \\
\hline Vollständigkeit & $\begin{array}{l}\text { Empfindlich auf äußere } \\
\text { Bedingungen } \\
\text { (Witterung,...) }\end{array}$ \\
$\begin{array}{l}\text { „Verhältnismäßig Kosten- } \\
\text { günstig“ }\end{array}$ & \\
\hline $\begin{array}{l}\text { Bildinformation als Zusatz } \\
\text { zur Geometrieinformation }\end{array}$ \\
\hline
\end{tabular}


Abb. 1: Gegenüberstellung gängiger Verfahren nach Genauigkeit und Eignung zur Erzeugung definierter Ergebnisse (nach [8])

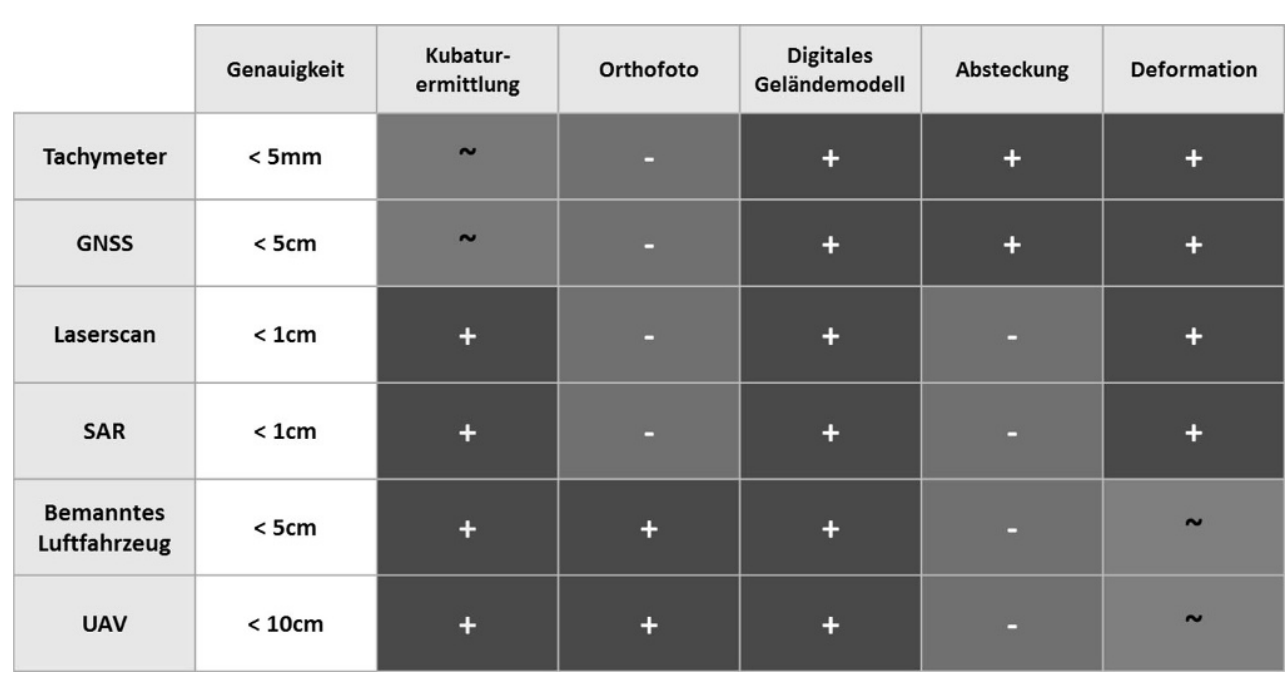

vor das vielseitigste Instrument ist und grundsätzlich fast alle anfallenden Aufgaben erfüllbar sind. Insbesondere da ein "Ortholuftbild“ eindeutig als nicht unbedingt notwendiges Ergebnis betrachtet werden kann.

Aus der Zusammenstellung ergibt sich jedoch auch, dass eine optimale Erfüllung aller Aufgaben durch eine Kombination verschiedener Verfahren zu erreichen ist. Nicht jedes Verfahren ist für jede Aufgabe geeignet, und ebenso können nicht alle Anforderungen von einer Lösung in optimaler Qualität erfüllt werden.

Betrachtet man in diesem Zusammenhang auch die aktuellen Trends in den technologischen Entwicklungen, so ist eine ganz klare Tendenz hin zur gemeinsamen Nutzung verschiedener Sensorik erkennbar. In Videotachymetern werden die Eigenschaften eines hochgenauen Theodoliten bzw. einer Totalstation mit der photogrammetrischen Aufnahme verknüpft. Das terrestrische Laserscanning wird in den meisten Fällen ebenfalls mit Kameras kombiniert. Und vor allem im Bereich der autonomen „mobile mapping“ Systeme wirken nahezu alle Komponenten zusammen. Basierend auf bestehenden Lösungen werden sozusagen neue „Typen von Vermessungsinstrumenten bzw. Plattformen" entwickelt, die zusehends stärker auch in die markscheiderische Praxis drängen werden, wie es am Beispiel von mit Kameras ausgerüsteten UAVs bereits sehr deutlich wird.

Vor diesem Hintergrund muss jedoch auch die Datenverarbeitung eine ähnlich schnelle Entwicklung nehmen. Bei immer neuen Möglichkeiten zur immer umfassenderen Datenakquisition gewinnt die Frage der Datenhaltung und besonders auch die Integration in gewohnte Arbeitsabläufe (Erzeugung gängiger und rechtlich notwendiger Produkte aus neuen Datenquellen) enorm an Bedeutung, wo mit hoher Sicherheit der größte zukünftige Forschungsbedarf gegeben ist.

Funding. Open access funding provided by Montanuniversity Leoben.

Open Access Dieser Artikel wird unter der Creative Commons Namensnennung 4.0 International Lizenz (http://creativecommons.org/licenses/ by/4.0/deed.de) veröffentlicht, welche die Nutzung, Vervielfältigung, Bearbeitung, Verbreitung und Wiedergabe in jeglichem Medium und Format erlaubt, sofern Sie den/die ursprünglichen Autor(en) und die Quelle ordnungsgemäß nennen, einen Link zur Creative Commons Lizenz beifügen und angeben, ob Änderungen vorgenommen wurden.

\section{Literatur}

1. Bundesgesetz über mineralische Rohstoffe (Mineralrohstoffgesetz MinroG), BGBI. I Nr. 38/1999

2. Mayer, G.; Pilgram, R.: Neue Entwicklungen in der Tagebauvermessung. In: Sroka, A. (Hrsg.): Schriftenreihe des Instituts für Markscheidewesen und Geodäsie, (2013-1), Nossen: Wagner Digitaldruck u. Medien $\mathrm{GmbH}, 2013$, S. 36-48

3. Verordnung des Bundesministers für Wirtschaft, Familie und Jugend über Vermessungen beim Bergbau, das Bergbaukartenwerk und die Erfassung von Bodenbewegungen 2013 (Markscheideverordnung 2013), BGBI. II Nr. 437/2012

4. Kahmen, H.: Angewandte Geodäsie: Vermessungskunde, 20., völlig neu bearbeitete Auflage, Berlin: Walter de Gruyter \& Co, 2006

5. Hofmann-Wellenhof, B.; Kienast, G.; Lichtenegger, H.: GPS in der Praxis, Wien, New York: Springer, 1994

6. Hanssen, R.F.: Radar Interferometry Data. Interpretation and Error Analysis, Dordrecht, Boston, London: Kluwer Academic Publishers, 2001

7. Pilgram, R.; Mayer, G.; Kremser, F.: Die messtechnische Erfassung von Bodenbewegungen an Hängen und Böschungen, BHM Bergund Hüttenmännische Monatshefte 156 (2011), Heft 4, S. 131-141

8. Tscharf, A.: Potentiale und Herausforderungen neuer Vermessungstechnologien im Bergbau - Der Einsatz unbemannter Flugkörper zur Erfassung von Geodaten, BHM Berg- und Hüttenmännische Monatshefte 161 (2016), Heft 10, S. 481-487 